\title{
Corrigendum
}

A matrix transfer function for an optical fibre based on coupled power theory

\section{P. RODHE}

Institute of Optical Research, S-100 44 Stockholm, Sweden

Opt. Quant. Elect. 13 (1981) 175-8.

On page 177, 12 lines from the top of the text, the words 'former' and 'latter' should be transposed. The correct sentence should read: 'This is explained by the fact that in the former the contribution from the more attenuated mode dominates over a longer distance than in the latter before a steady state is established'.

The author's name was misspelt in the paper: he is P. Rodhe not P. Rohde. 